\title{
Annexe : le marché des lubrifiants et des biolubrifiants
}

\section{Étienne POITRAT}

\section{ADEME Département Bioressources \\ <etienne.poitrat@ademe.fr>}

La définition de la nature de l'ensemble des lubrifiants et biolubrifiants correspond aux huiles de base utilisées et qui peuvent être classées selon les cinq familles suivantes (figure 1):

- huiles végétales non modifiées,

- esters oléochimiques,

- esters synthétiques,

- hydrocarbures synthétiques,

- huiles minérales.

La consommation française de lubrifiants était de 841000 tonnes en 2002, dont près de $45 \%$ sont des huiles moteur. Elle représentait $17,5 \%$ de la consommation européenne et avait diminué d'environ $3 \%$ par rapport à 1995, ceci étant dû à la régression des huiles moteur. Le marché se répartit de la façon suivante (tableau 1).

En France, le marché des lubrifiants biodégradables (origine végétale ou pétrolière) est inférieur à 3500 tonnes et celui des biolubrifiants (origine végétale, biodégradables ou pas) est inférieur à 1000 tonnes (tableau 2).

Des perspectives de développement importantes peuvent être envisagées, lorsque le bénéfice environnemental et sanitaire est immédiat dans les utilisations à huiles perdues ou à risque de pertes accidentelles dans l'environnement, et pour les applications dans les industries agroalimentaires. En outre, les lubrifiants d'origine végétale présentent des performances techniques au moins égales aux huiles synthétiques. Les critères d'écocompatibilité suivants sont reconnus et fréquemment utilisés: biodégradabilité, toxicité humaine, écotoxicité (aquatique ou terrestre), bioaccumulation.

Mais il n'existe pas de réglementation européenne qui imposerait des critères d'écocompatibilité (biodégradabilité, nontoxicité, non-écotoxicité, origine de l'huile de base, volatilité, présence de substances dangereuses, etc.) pour une application en lubrification.

II reste à appliquer une véritable stratégie de développement et de pénétration du marché des lubrifiants qui peut être aidée par les dispositions de management environnemental (par exemple: certification ISO 14001) et des démarches-qualité. Des directives européennes devraient prochainement être appliquées en ce qui concerne la toxicité et la dangerosité des produits.

\section{RÉFÉRENCE}

1. Bio Intelligence Service $S A$ et AriaConsult pour I'Ademe. Etude du marché français des biolubrifiants, Février 2004. Synthèse de l'étude disponible sur le site www.ademe.fr.

Tableau 1. Marché des lubrifiants par famille d'application.

\begin{tabular}{|c|c|c|c|c|}
\hline \multirow[t]{2}{*}{ Familles d'application } & \multirow[t]{2}{*}{$\begin{array}{c}\text { Principaux secteurs } \\
\text { utilisateurs }\end{array}$} & \multirow{2}{*}{$\begin{array}{c}\text { Consommation } \\
1995 \\
\text { France }\end{array}$} & \multicolumn{2}{|c|}{$\begin{array}{c}\text { Consommation } \\
2002\end{array}$} \\
\hline & & & France & $\begin{array}{l}\text { Europe } \\
\text { des } 15\end{array}$ \\
\hline Huiles moteur & Automobile, bateaux, avions & $\begin{array}{c}429039 t \\
49,5 \%\end{array}$ & $\begin{array}{c}377359 \mathrm{t} \\
44,9 \%\end{array}$ & $\begin{array}{c}1910809 \mathrm{t} \\
39,8 \%\end{array}$ \\
\hline $\begin{array}{l}\text { Engrenages et } \\
\text { transmission }\end{array}$ & Automobile, industrie & $\begin{array}{c}176597 \mathrm{t} \\
20,4 \%\end{array}$ & $\begin{array}{c}187779 \mathrm{t} \\
22,3 \%\end{array}$ & $\begin{array}{c}1130002 \mathrm{t} \\
23,5 \%\end{array}$ \\
\hline Graisses & Automobile et Industrie & $\begin{array}{c}24020 \mathrm{t} \\
2,8 \%\end{array}$ & $\begin{array}{c}23526 \mathrm{t} \\
2,8 \%\end{array}$ & $\begin{array}{c}132231 \mathrm{t} \\
2,8 \%\end{array}$ \\
\hline Travail des métaux & Métallurgie & $\begin{array}{l}70696 \mathrm{t} \\
8,2 \%\end{array}$ & $\begin{array}{l}73165 \mathrm{t} \\
8,7 \%\end{array}$ & $\begin{array}{c}322535 \mathrm{t} \\
6,7 \%\end{array}$ \\
\hline Huiles surraffinées & $\begin{array}{l}\text { Turbines, transformateurs, } \\
\text { huiles de chaînes }\end{array}$ & $\begin{array}{c}21146 \mathrm{t} \\
2,4 \%\end{array}$ & $\begin{array}{c}23957 \mathrm{t} \\
2,8 \%\end{array}$ & $\begin{array}{c}144407 \mathrm{t} \\
3,0 \%\end{array}$ \\
\hline 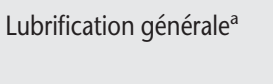 & $\begin{array}{c}\text { Démoulage et décoffrage, } \\
\text { mouvements }\end{array}$ & $\begin{array}{c}59377 \mathrm{t} \\
6,8 \%\end{array}$ & $\begin{array}{c}49888 \mathrm{t} \\
5,9 \%\end{array}$ & $\begin{array}{c}395610 \mathrm{t} \\
8,2 \%\end{array}$ \\
\hline $\begin{array}{l}\text { Huiles de procédés } \\
\text { + huiles blanches }\end{array}$ & Agroalimentaire, pharmacie & $\begin{array}{c}85293 \mathrm{t} \\
9,9 \%\end{array}$ & $\begin{array}{c}105682 \mathrm{t} \\
12,6 \%\end{array}$ & $\begin{array}{c}763533 \mathrm{t} \\
15,9 \%\end{array}$ \\
\hline Total & & $\begin{array}{c}86 \mathbf{1 6 8} \mathbf{t} \\
100 \%\end{array}$ & $\begin{array}{c}841356 \mathbf{t} \\
100 \%\end{array}$ & $\begin{array}{c}4799127 t \\
100 \%\end{array}$ \\
\hline
\end{tabular}

Source : Centre professionnel des lubrifiants (1995) et Europalub (2002)

a Lubrification générale regroupe : huiles pour compresseurs, huiles pour mouvements toutes viscosités, huiles pour outils pneumatiques, glissières et huiles pour le graissage perdu, huiles industrielles à usage non-lubrifiant.

Tableau 2. Estimation du marché français en 2003 (ordres de grandeur).

\begin{tabular}{|lcc|}
\hline & $\begin{array}{c}\text { Total Végétal (t) } \\
\text { (biodégradables } \\
\text { ou pas) }\end{array}$ & $\begin{array}{c}\text { Total Biodégradable (t) } \\
\text { (origine végétale ou pétrochimique, } \\
\text { hors origine animale) }\end{array}$ \\
\hline Huiles de chainne & $<200$ & $<300$ \\
Huiles hydrauliques & $<400$ & $<1000$ \\
Huiles moteur 2 temps nautique & - & $<500$ \\
Démoulage et décoffrage & $<50$ & $<200$ \\
Forage pétrolier & $<200$ & $<500$ \\
Divers (graissage, engrenage, & $<150$ & $<1000$ \\
transmission, etc.) & $<\mathbf{0 0 0}$ & $<\mathbf{3 0 0}$ \\
Total & $<\mathbf{1 0 0 0}$ & \\
\hline
\end{tabular}

Ces chiffres n'incluent pas les additifs de toutes natures dont les additifs sulfurisés (par exemple à base d'huile de café) à partir d'huile de lin ou de colza, utilisés dans le travail des métaux, qui représenteraient 500 à 600 tonnes.

\begin{tabular}{|c|c|l|c|c|}
\hline \multicolumn{2}{|c|}{$\begin{array}{c}\text { Matière première d'origine } \\
\text { végétale/animale }\end{array}$} & \multicolumn{3}{c|}{ Matière première d'origine pétrolière } \\
\hline $\begin{array}{c}\text { Huiles végétales } \\
\text { non modifiées }\end{array}$ & $\begin{array}{c}\text { Esters } \\
\text { oléochimiques }\end{array}$ & Esters synthétiques & $\begin{array}{c}\text { Hydrocarbures } \\
\text { synthétiques }\end{array}$ & $\begin{array}{c}\text { Huiles } \\
\text { minérales }\end{array}$ \\
\hline & \multicolumn{3}{|c|}{ Huiles de synthèse } & \\
\hline
\end{tabular}

Figure 1. Les lubrifiants et biolubriants. 